\title{
Pareto Set-based Ant Colony Optimization for Multi-Objective Surgery Scheduling Problem
}

\author{
Xiang Wei ${ }^{1, *}$ and Gino Lim $^{2}$ \\ ${ }^{1}$ Faculty of Mechanical Engineering and Mechanics, Ningbo University, Ningbo, 315211, P.R. China; ${ }^{2}$ Department of \\ Industrial Engineering, The University of Houston, Houston, TX, 77204, USA
}

\begin{abstract}
Surgery scheduling determines the individual surgery's sequence and assigns required resources. This task plays a decisive role in providing timely treatment for the patients while ensuring a balanced hospital resources' utilization. Considering several real life constraints associated with multiple resources during the complete 3-stage surgery flow, a surgery scheduling model is presented with multiple objectives of minimizing makespan, minimizing overtime and balancing resource utilization. A Pareto sets based ant colony algorithm with corresponding ant graph, pheromone setting and update, and Pareto sets construction is proposed to solve the multi-objective surgery scheduling problem. A test case from MD Anderson Cancer Center is built and the scheduling result by three different approaches is compared. The case study shows that the Pareto set-based ACO for multi-objective proposed in this paper achieved good results in shortening total end time, reducing nurses' overtime and balancing resources' utilization in general. It indicates the advantage by systematically surgery scheduling optimization considering multiple objectives related to different shareholders.
\end{abstract}

Keywords: Ant colony optimization, multi-objective optimization, operating room management, pareto set, surgery scheduling.

\section{INTRODUCTION}

One of the main challenges in health care systems in recent years is to deliver high quality service under limited available resources. With the increase of aging population, social demands for surgical service have been constantly increased [1]. As a vital hospital component, the operating room (OR) division accounts for approximately more than $40 \%$ of a hospital's total revenues and expenses [2]. Hence, it is essential to improve patient flow and optimize OR management in order to provide timely treatments for the patients and to maximize utilization of the available resources.

Surgery scheduling plays a crucial role in the OR management. It determines the operation time and allocates the resources to surgeries to be performed in different surgical specialties over a schedule period. The overall surgery process involves three main stages, i.e. pre-surgery, surgery, and post-surgery. The resources required to perform a surgery comprise personnel (surgeons, anaesthetists, nurses, etc.) as well as facilities (specialized equipment, pre-operative holding units (PHUs), multiple ORs, post anaesthesia care units (PACUs), and intensive care units (ICUs)). Moreover, all the required resources have to be occupied simultaneously. Surgery scheduling problem has been a widely studied topic and there exists a vast amount of literature in the medical and operation research. Reviews about OR planning and scheduling have been conducted most recently [3-6]. They provided detailed classifications of researches based on problemspecified, operations research methodology, and decision levels.
Multiple surgery flow stages and multiple resources involved in OR management not only increases the complexity of surgery scheduling problem, but also calls for the more comprehensive evaluation of scheduling result. The evaluation on the efficiency of surgery scheduling should consider several performances including patients' satisfaction, staffs satisfaction and OR management effectiveness. The patients' satisfaction depends directly on the timely surgery arrangement, less waiting time, and quality treatment. Staffs satisfaction is usually expressed as less overtime and a more balanced task. The effectiveness of OR management can be further described as management cost and resources' utilization. Therefore, it is necessary to take multiple objectives into account for surgery scheduling. Several surgery scheduling problems described in the literature consider multiple objectives. Ogulata and Erol developed a set of hierarchical multiple criteria mathematical programming models to generate weekly operating room schedules. The objectives considered in this study are maximum utilization of operating room capacity, balanced distribution of operations among surgeon groups and minimization of patient waiting times [7]. Cardoen et al. present a multi-objective optimization model for scheduling individual cases in the surgical daycare center of a large Belgian hospital [8]. Six different objectives (children and prioritized patients as early as possible, patients having a large travel distance as much as possible before a particular hour, minimizing overtime in recovery and levelling bed occupancy in both recovery phase 1 and recovery phase 2) are considered in the model. Beliën et al. presented a decision support system to develop master surgery schedules. The system is built on different optimization procedures that aim at levelling the resulting bed occupancy, concentrating surgeons of the same group in the same rooms, 
Table 1. The notations.

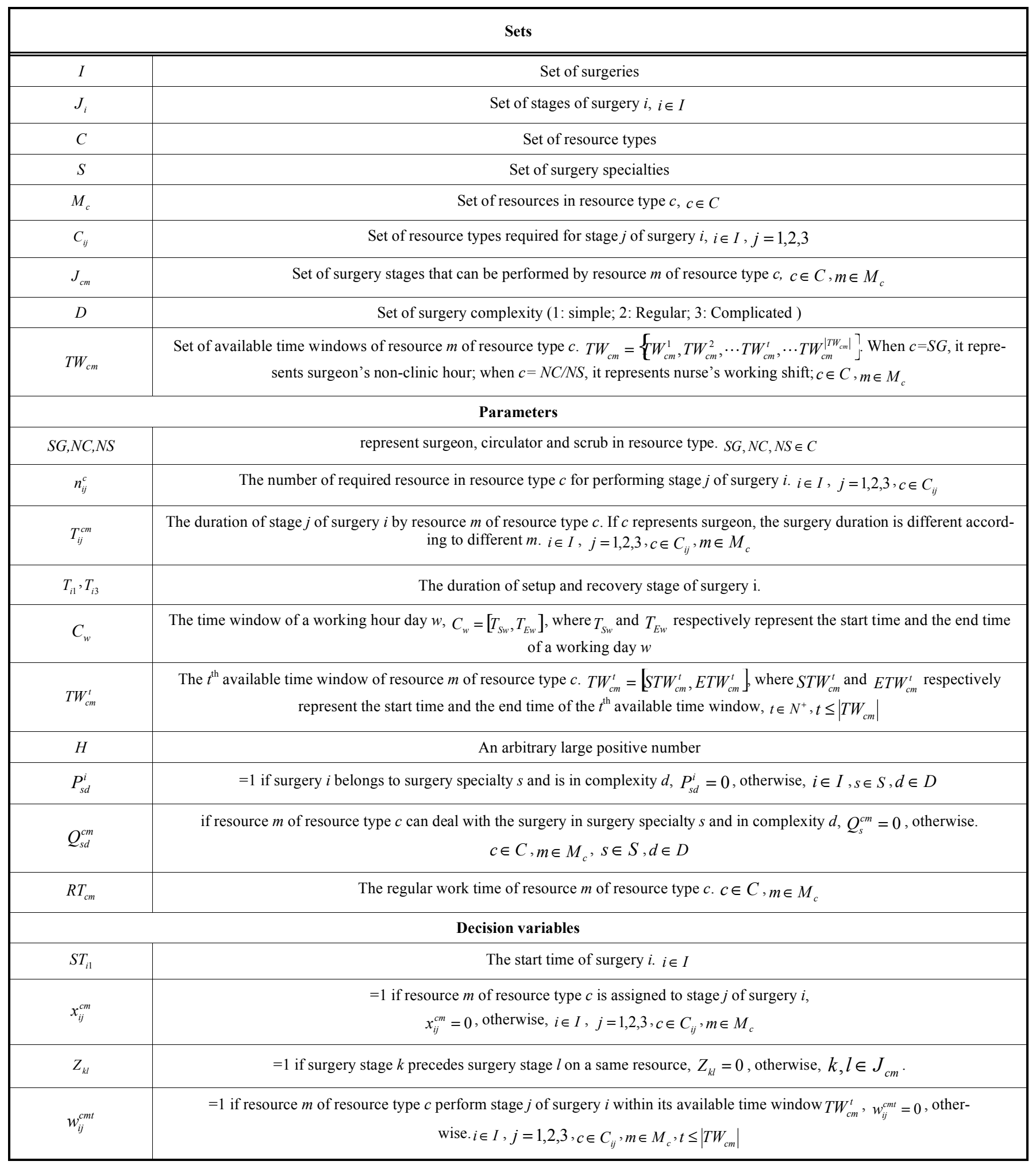

and keeping the schedules consistent from week to week [9]. Jeric and Figueira addressed a multi-objective scheduling problem in a Croatian hospital and formulated it as a BIP model. Considering the discrete search space, numerous variables, constraints and multiple objectives, they proposed several meta-heuristics, i.e. a variable neighborhood search, scatter search and a non-dominated sorting genetic algorithm for scheduling medical treatments [10]. Although not necessarily, the performances often conflict with each other, meaning that building an optimal schedule with respect to one objective goes at the cost of the other objectives. Further more, the combinatorial nature and the nonlinearity in con- 
straints of the surgery scheduling problem make it extremely difficult to optimize. In this paper, a Pareto Set-based ant colony algorithm is proposed to solve such multi-objective surgery scheduling problem. Most literature researches solved multi-objective surgery scheduling problem by weighting objectives. Our approach integrates the Pareto set solutions to the ACO so as to make it more efficient even when the conflicting multiple objectives exist.

The rest of the paper is organized as follows. In Section 2 the multi-objective scheduling problem is outlined. In Section 3 we introduce the Pareto Set-based ACO algorithm and present the detail mechanisms in such approach. In Section 4 , we provide the computational experiments to validate and evaluate our approach. We close in Section 5 with summary and suggestions for future research.

\section{MULTI-OBJECTIVE SURGERY SCHEDULING MODEL}

We assume that there is a set of surgeries, represented as $I$, to be performed in an operating system with different types of resources (including both personnel resources and facilities associated with surgery groups). Each surgery has its surgery demand and resource demand. Surgery demand determines a surgery can only be performed by several surgeons in a special surgery group, and its operating time varies by surgeon. Resource demand indicates all resources required for the complete stages of a surgery. Whether a stage can start successfully is restricted not only by the variety of resources, but also by the performance of each previous stage. The scheduling goal is to select the best resources, determine the various surgeries' operating sequences, and get the shortest makespan, the minimum overtime and the balanced utilization, considering the diversities of types and quantity of resources and the mutual constraint of their available time.

The notations used for our model are listed in Table 1. The multi-objective mathematic model is described as following:

The objective function is to minimize the three objective $f_{1}, f_{2}$ and $f_{3}$.

$$
\min \vec{F}=\left(f_{1}, f_{2}, f_{3}\right)
$$

The three objective functions include:

1) The $1^{\text {st }}$ objective is to minimize the time to finish all surgeries, so-called makespan.

$f_{1}=\min \max _{i \in I} E T_{i 3}$

2) The $2^{\text {nd }}$ objective function is to minimize the variation coefficient of resources working time (VCWT).

$f_{2}=\min \max _{c \in C} C V_{c}$

$C V_{c}$ is the VCWT of resources in resource type $c$ and is defined as the ratio of the standard deviation to mean as shown in equation (4). It is used to evaluate the balance of resource utilization.

$C V_{c}=\sigma_{c} / \mu_{c}$
$\mu_{c}=\frac{1}{\left|M_{c}\right|} \sum_{m \in M_{c}} \sum_{j \in J_{i}} \sum_{i \in I} T_{i j}^{c m} x_{i j}^{c m}$

$\sigma_{c}=\sqrt{\frac{1}{\left|M_{c}\right|} \sum_{m \in M_{c}}\left(\sum_{j \in J_{i}} \sum_{i \in I} T_{i j}^{c m} x_{i j}^{c m}-\mu_{c}\right)^{2}}$

3) The $3^{\text {rd }}$ objective is to minimize the total overtime of all resources:

$f_{3}=\min \left\{\sum_{c \in C} \sum_{m \in M_{c}}\left(\max _{i \in I, j \in J_{i}}\left(E T_{i j} \cdot x_{i j}^{c m}\right)-R T_{c m}\right)\right\}$

The constraints include:

1) The end time of stage $j$ of surgery $i$ is determined by the start time and the maximum duration of all assigned resources.

$E T_{i j}=S T_{i j}+\max _{c \in C_{i j}, m \in M_{c}}\left\{T_{i j}^{c m} \cdot x_{i j}^{c m}\right\}, i \in I, j=1,2,3$

2) For any two consecutive stages $j$ and $j+1$ of a surgery $i, j+1$ starts immediately when $j$ has finished its processing step.

$E T_{i j}=S T_{i(j+1)}, i \in I, j=1,2$,

3) Any two stages of a surgery can not to be performed at the same time.

$E T_{i q}-E T_{i p} \geq T_{i q}^{c m}, p<q \in J_{i}, i \in I$

4) A resource can only be assigned to one surgery stage at a time.

$$
\begin{aligned}
& E T_{e l}-E T_{i k}+H\left(1-Z_{k l}\right) \geq T_{e l}^{c m}, k, l \in J_{c m}, i, e \in I, \\
& E T_{i k}-E T_{e l}+H Z_{k l} \geq T_{i k}^{c m}, k, l \in J_{c m}, i, e \in I, i \neq e,
\end{aligned}
$$

5) The surgery must be performed within the same working day. i.e. $S T_{i 1}, E T_{i 3} \in C_{w}$

$T_{S w} \leq S T_{i 1} \leq T_{E w}-T_{i 1}-T_{i 3}-\max \left\{T_{i 2}^{c m} \cdot x_{i j}^{c m}\right\}$,

$c \in C_{i j}, m \in M_{c}, i=1,2, \cdots n, w \in N^{+}$

6) The exactly required number of resources in resource type $c$ is assigned to perform stage $j$ of surgery $i$.

$\sum_{m \in M_{c}} x_{i j}^{c m}=n_{i j}^{c}, c \in C_{i j}$

7) The surgery must be started and finished within the assigned resource's available time window.

$$
\begin{aligned}
& S T_{i j} \geq x_{i j}^{c m} \cdot \sum_{t=1}^{\left|T W_{c m}\right|}\left(w_{i j}^{c m t} \cdot S T W_{c m}^{t}\right) \\
& E T_{i j} \leq x_{i j}^{c m} \cdot \sum_{t=1}^{\left|T W_{c m}\right|}\left(w_{i j}^{c m t} \cdot E T W_{c m}^{t}\right), \\
& i \in I, j=1,2,3, c \in C_{i j}, m \in M_{c}
\end{aligned}
$$

8) The resource (here mainly refer to surgeon, circulator and scrub) assigned to a surgery must have the ability in re- 
quired surgery specialty and required qualification of dealing with surgery complexity.

$$
\begin{aligned}
& x_{i j}^{c m} \leq \sum_{d \in D} \sum_{s \in S} P_{s d}^{i} \cdot Q_{s d}^{c m}, i \in I, j=1,2,3, \\
& c \in C_{i j} \cap\{S G, N C, N S\}, m \in M_{c}
\end{aligned}
$$

10) Followings are binary variables

$$
\begin{aligned}
& x_{i j}^{c m} \in\{0,1\}, i \in I, j=1,2,3, c \in C_{i j}, m \in M_{c} \\
& z_{k l} \in\{0,1\}, k, l \in J_{c m}, \\
& w_{i j}^{c m t} \in\{0,1\}, i \in I, j=1,2,3, c \in C_{i j}, m \in M_{c}, t \leq\left|T W_{c m}\right|
\end{aligned}
$$

\section{PARETO SET BASED ANT COLONY ALGO- RITHM FOR MOB SURGERY SCHEDULING}

The combinatorial nature and the nonlinearity in constraints of the above surgery scheduling problem make obtaining an optimal scheduling result challenging. Instead, we aimed at a meta-heuristic approach for sub-optimal solution and developed a Pareto set based ACO algorithm to solve the multi-objective surgery scheduling problem.

Since the complete surgery scheduling determines both the resources allocation for each of the surgery stages and the sequencing of surgeries in time period, a two-level ant graph is designed. In our previous research, an ant colony algorithm with such two-level ant graph was proposed for solving the surgery scheduling problem with single objective of makespan (Later in section 4, we name it as ACO-SOB) [11]. The outer level graph is defined as a surgery graph. The nodes in the surgery graph represent the surgeries, and the directional arc indicates the precedence sequence. The ant foraging path is the scheduling sequence of the surgeries [12]. The inner level graph is defined as resources graph. The inner-graph node represents the total available resources of the OR management system along the three-stage surgery procedure. Ant foraging path in inner level graph determines the resources selection for each specific stage during a surgery.

\subsection{Pheromone definition and update strategy}

As to ACO for single objective optimization, the pheromone definition and update strategy are designed to efficiently record and enhance the pheromone to a specific (an optimal) solution [13]. However, in the multi-objective optimization, there may no longer exist such optimal solution, but a Pareto set of solutions. Therefore, a specific pheromone setting, called single-path-multi-pheromone (SPMP), and the corresponding pheromone update strategies are introduced to take into account the impact of several Pareto optimal solutions due to multiple objectives. SPMP allows laying multiple pheromone value on a single ant path according to the number of objectives. A pheromone vector consists of three pheromone values which are associated to three objectives $(r=1,2,3)$ is defined on each path.

In outer ant graph, the sequence-related pheromone $\left(\tau_{i j}\right)$ is defined in equation (21) to indicate the strength of sequence from node $i$ to node $j$. Its value is determined by the individual objective strength $\tau_{i j}^{r}$ and the associated weights $\omega_{r}, r=1,2,3$.

$\tau_{i j}=\sum_{r=1}^{3} \omega_{r} \tau_{i j}^{r}$

$\tau_{i j}^{r}=(1-\rho) * \tau_{i j}^{r}+\Delta \tau_{i j}^{r}\left(s_{r}\right)$

$\Delta \tau_{i j}^{r}\left(s_{r}\right)=\left\{\begin{array}{l}\frac{Q_{r}}{L_{s_{r}}^{r}}, \text { if ant } s_{r} \text { goes through }(i, j) \text { in this iteration } \\ 0, \quad \text { otherwise }\end{array}\right.$

As to the pheromone update strategy (equation (22) and (23)), an iteration-Pareto-optimal (IPO) update strategy is adopted to cluster ants to paths with Pareto optimal solutions. We set $Q_{r}$ as the pheromone strength vector associated with three objectives. $L_{s}^{r}$ is the $r^{\text {th }}$ objective value of a solution $s$. Only the pheromone value associated to the individual best objective is reinforced, the other two pheromone value in vector keep unchanged. However, evaporation will be happened for all pheromones in pheromone vector, $\rho$ denotes the pheromone evaporation rate.

The inner surgery-related pheromone $\left({ }^{I n} \tau_{c m}^{i}\right)$ is defined to associate surgery $i$ with resource $m$ of resource type $c$. Its detail definition and update strategy are the same to above sequence-related pheromone, and are described in equation (24) to (26).

$$
\begin{aligned}
& { }^{I n} \tau_{c m}^{i}=\sum_{r=1}^{3} \omega_{r}{ }_{r}^{I n} \tau_{c m}^{i r} \\
& { }^{I n} \tau_{c m}^{i r}=(1-\rho) \cdot{ }^{I n} \tau_{c m}^{i r}+\Delta^{I n} \tau_{c m}^{i r}\left(s_{r}\right) \\
& \Delta^{I n} \tau_{c m}^{i r}\left(s_{r}\right)= \begin{cases}\frac{Q_{r}}{L_{s}^{r}}, & \text { if ant } s_{r} \text { visits resource (c, m) for surgery } i \\
0, & \text { otherwise. }\end{cases}
\end{aligned}
$$

The inner resource-related pheromone ${ }^{I n} \lambda_{m}^{k}$ is defined for recording the information related to resource utilization during an ant constructing inner resource allocation solution. It starts to be effective as long as an ant enters to inner resource graph, and ends to be empty when an ant goes out to outer graph. In any surgery stage, once a resource is selected, its opportunity to be selected by other surgery should be reduced so as to balance the resource utilization. Therefore, it is updated locally after visiting each node (i.e. on arc-step completion) as equation (27), where $q_{0}$ is the decremented pheromone value. By local pheromone updating, the possibility of ants crawling through the same path decreases, thus it can effectively avoid the uneven utilization of resources.

${ }^{I n} \lambda_{m}^{k}={ }^{I n} \lambda_{m}^{k}-q_{0}$

\subsection{Construct Pareto Set}

Individual ant traverses the two-level ant graph to build a feasible schedule solution which includes both the surgeries' sequence and the resources allocation for individual surgery. The detail probabilistic transition rule used in ant solution construction can be found in our previous work [11]. Usually 
in multi-objective optimization, typically there exists no feasible solution that minimizes all objective functions simultaneously. Therefore, Pareto optimal solutions, i.e. solutions that cannot be improved in any of the objectives without impairment in at least one of the other objectives, are proposed.

Surgery scheduling problem in this work is described as a multi-objective optimization problem with three objectives: $\min \vec{F}=\left(f_{1}, f_{2}, f_{3}\right)$. Assume $S$ is the feasible solution set. In mathematical terms, a feasible solution $s_{1} \in S$ is said to dominate another solution $s_{2} \in S$, represented as $\left(s_{1} \succ s_{2}\right)$, if 1) $f_{i}\left(s_{1}\right) \leq f_{i}\left(s_{2}\right), i \in\{1,2,3\}$ and 2) $f_{j}\left(s_{1}\right)<f_{j}\left(s_{2}\right), \exists j \in\{1,2,3\}$. Solution $s_{1}$ is called Pareto optimal, if there does not exist another solution that dominates it. The set of Pareto optimal is often called the Pareto set.

Pareto set based ant colony algorithm for multi-objective optimization (ACO-MOB) is to construct such Pareto set for each ant cycle, to keep update Pareto set along with the iteration, and to finally approach to Pareto optimal set. The final decision can be the one based on decision maker's preference in such Pareto optimal set. Assume a feasible solution set $S$ with $N$ solution, and a Pareto set $P S$, initially set as $n i l$. A challenge-like algorithm is designed in ACO to construct Pareto set as following:

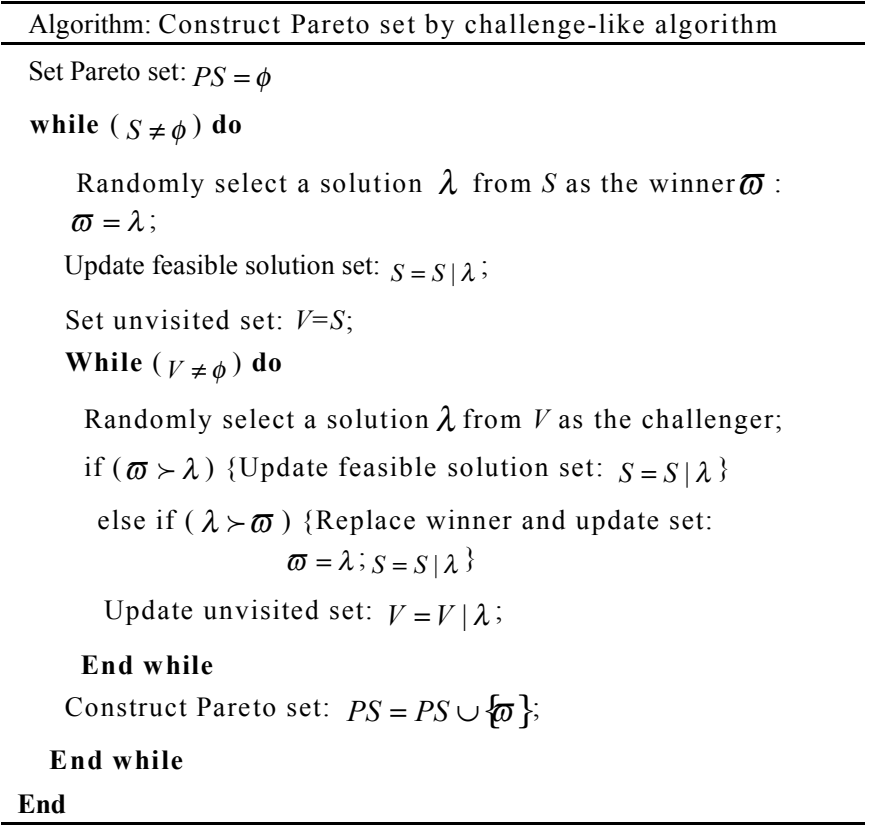

\subsection{ACO-MOB Algorithm Description}

The detailed procedure of the proposed ACO-MOB algorithm for the multi-objective surgery scheduling problem is explained as follows:

\begin{tabular}{l}
\hline Algorithm: the Pareto set based ACO-MOB for surgery scheduling \\
\hline Step1: Build an ant graph model \\
While (iteration termination condition not met) do \\
Step 2: Put $m$ ants on arbitrary node \\
Step 3: Initialize pheromone trial
\end{tabular}

Step 4: Construct a feasible solution set $S$ by ants traversing outer and inner ant graph

While (ant termination condition not met, $\mathrm{k}<\mathrm{m}$ ) do

Step 4.1: Initialize solution $t a b u=\boldsymbol{\phi}$; and surgeries set $I$

Step 4.2: Construct an ant solution by visiting a node $i$ in outer graph based on the state transition rule; then update tabu $=t a b u \cup\left\{I_{i}\right\}$ and $I=I \backslash\left\{I_{i}\right\}$

Step 4.3: Ant enters into the inner graph, construct resource set G

Step 4.4: Construct a resource allocation solution

for each demanding resource types $c$ do

Step 4.4.1: Construct an ant solution by visiting a node in inner graph based on the state transition rule

Step 4.4.2: Local update inner resource-related pheromone

End for

Step 4.5: Decoding \& Update resources’ time window

Step 4.6: if $I \neq \phi$, go to Step 4.1.

else add ant $k$ 's solution $t a b u$ to feasible solution set $S$ : $S=t a b u \cup S$

End While

Step 5: Construct a Pareto set $P S_{\text {now }}$ by challenge-like algorithm.

Step 6: Form an iteration feasible set $S_{\text {iteration }}$ by combining Pareto set $P S_{n o w}$ with previous iteration Pareto set $P S_{\text {iteration }}$ :

$S_{\text {iteration }}=P S_{\text {iteration }} \cup P S_{\text {now }}$

Step 7: Construct a final iteration Pareto set $P S_{\text {iteration }}$ by re-apply challenge-like algorithm

Step 8: Update sequence-related and surgery-related pheromone in both outer and inner ant graph based on IPO update strategy.

End While

Step 9: Select a solution $S^{*}$ from a final Pareto set $P S_{\text {iteration }}$ according to decision maker's preference.

End

\section{EMPIRICAL STUDY}

The proposed ACO-MOB algorithm is implemented with Matlab and is run on a PC running Windows XP with Intel Core5@2.79GHz and 3GB of memory.

\subsection{Test Case Description}

Data for the experiment was extracted from MD Anderson Cancer Center, one of the world's most respected cancer treatment facilities. A daily scheduling data set was built around 28 cases in 17 ORs to be staffed by 23 RNs and 14 scrubs each working one of the five shifts starting at 6:30 AM and ending at 11:30 PM. Surgical related information and daily roster information are given in reference [14].

Four performance measurements: end time, VCWT, maximum overtime of nurses, and total overtime of nurses are used for comparison. End time, i.e. the finishing time of all 28 surgeries to be scheduled, is to measure the efficiency of the scheduling. VCWT is defined as the ratio of the standard deviation to mean and it is used to evaluate the balance of resource utilization. The smaller value in VCWT means the better utilization among resources. A zero value means a fully balanced assignment in resources utilization. Since in real-life OR management, nurses are assigned to work in different shifts, therefore nurses are further divided into groups according to shifts when calculating the VCWT of 
Table 2. The optimal ACO-MOB parameters.

\begin{tabular}{|c|c|c|c|c|c|c|c|}
\hline $\mathbf{M}$ & $\alpha$ & $\beta$ & $\rho$ & $\boldsymbol{Q}$ & $\boldsymbol{Q}_{0}$ & $\boldsymbol{N} C_{-} \boldsymbol{m a x}$ & $\boldsymbol{w 1 : w 2 : w 3}$ \\
\hline \hline 150 & 1 & 5 & 0.5 & {$[50,1.5,60]$} & 0.2 & 150 & $0.2: 0.2: 0.6$ \\
\hline
\end{tabular}

Table 3. Surgery scheduling results by ACO-MOB algorithm.

\begin{tabular}{|c|c|c|c|c|c|c|c|}
\hline 1 & 1 & 9 & 6 & 15 & 15 & 26 & 7 \\
\hline 3 & 12 & 29 & 32 & 17 & 7 & 27 & 33 \\
\hline 6 & 8 & 1 & 13 & 20 & 4 & 6 & 14 \\
\hline 7 & 14 & 23 & 21 & 21 & 3 & 26 & 24 \\
\hline 8 & 5 & 16 & 35 & 22 & 15 & 4 & 5 \\
\hline 12 & 17 & 34 & 8 & 26 & 1 & 10 & 17 \\
\hline 13 & 5 & 34 & 7 & 27 & 8 & 28 & 22 \\
\hline 14 & 9 & 18 & 12 & 28 & 16 & 29 & 32 \\
\hline
\end{tabular}

nurses. Overtime (OT) records the total additional time required in addition to the regular working hours and directly effects the cost of OR management. Such OT measurement is further evaluated by both the maximum OT among nurses and the total OT among nurses. An authorized overtime is an additional time that nurses can be assigned if a surgery is not finished by the end of the regular shift.

\subsection{The Optimal ACO-MOB Parameters Setting}

The basic ACO-MOB parameters include the number of ants $(\mathrm{m})$, pheromone factor $(\alpha)$, heuristic factor $(\beta)$, evaporate rate $(\rho)$, pheromone intensity $(\mathrm{Q})$, decremented pheromone value (q0), and weights of multiple objectives $\left(\omega_{r}\right)$. Those parameters have impact on the ant exploration and ant following known pheromone, which brings difference in algorithm's convergence and solution quality. Several experiments are built to identify the optimal parameters by different adjustments, and Table 2 lists the optimal ACOMOB parameters achieved.

\subsection{Computational Result Discussion}

A comparison experiment is built to evaluate the performance of three different scheduling approaches. These three approaches are receptively, the manual scheduling in MD Anderson Cancer Center (named as 'Manual'), the basic
ACO approach with single objective of makespan (named as 'ACO-SOB'), and the proposed ACO-MOB in this work.

The final surgery scheduling result with resources' assignment solved by our ACO-MOB is listed in Table 3 . The comparison results by three different scheduling approaches in several measurements are shown in Table 4. From Table 4, both ACO-MOB and ACO-SOB achieve a schedule with all 28 surgeries finished by 18:30 and has 1.5 hour reduction compared to manual scheduling. Such one and half hour reduction in end time can be a good proof that surgery scheduling by ACO has advantage in scheduling efficiency.

As to the resources utilization, except VCWT value in shift 3 nurse is 0 because of only one nurse on duty, the VCWT of other resources including ORs and nurses in shift1 and shift 2 all show varying improvements. The VCWT of ORs in ACO-SOB and ACO-MOB is 0.21 and 0.2 , which has a reduction around $36 \%$. Both VCWT of nurses in shift 1 and shift 2 by ACO approach have a much better value of $0.18(0.21)$ and $0.05(0.06)$ compared to those by manual scheduling of 0.35 and 0.37 . The improvement is $68 \%(63 \%)$ for shift 1 and $68 \%(63 \%)$ for shift 2 . There is not much difference in VCWT of nurses and ORs by ACO-MOB or ACO-SOB.

OT means the increasing of cost, which is not wanted in OR management. The third observation from comparison results is the improvement in OT measurements in both 
Table 4. Comparison on three approaches.

\begin{tabular}{|c|c|c|c|c|c|c|c|}
\hline & \multirow{3}{*}{ End Time } & \multicolumn{4}{|c|}{ Variation Coefficient of Resources' Working Time: VCWT } & \multirow{3}{*}{$\begin{array}{c}\text { Individual Nurse' } \\
\text { Maximum OT } \\
\text { (h) }\end{array}$} & \multirow{3}{*}{$\begin{array}{c}\text { Nurses' Total OT } \\
\text { (h) }\end{array}$} \\
\hline & & \multirow{2}{*}{ OR } & \multicolumn{3}{|c|}{ Nurse } & & \\
\hline & & & Shift 1 & Shift 2 & Shift 3 & & \\
\hline Manual & $19: 30$ & 0.33 & 0.57 & 0.16 & 0 & 12 & 12.5 \\
\hline ACO-SOB & $18: 30$ & 0.21 & 0.18 & 0.05 & 0 & 2.75 & 11 \\
\hline ACO-MOB & $18: 30$ & 0.20 & 0.21 & 0.06 & 0 & 2.75 & 7 \\
\hline
\end{tabular}

maximum OT of individual nurse and the total OT of all nurses. Individual nurse's maximum OT is reduced to 2.75hours and has a 9.25hours improvement compared to 12 hours in manual work. Total OT of nurses is also reduced from 12.5 hours to 11 hours by ACO-SOB, and to 7hours by ACO-MOB. It records around $36 \%$ improvement by comparing ACO-MOB with ACO-SOB.

In sum, the comparison result shows that the proposed Pareto set-based ACO algorithm for surgery scheduling has good performance on shortening total time and allocating resources for surgery scheduling.

\section{CONCLUSION AND ONGOING WORK}

We have developed a Pareto set-based ACO approach for solving multiple objective surgeries scheduling problem that arises in large operating suites. The problem is complicated because of involving the complete stages of surgery flow and multiple resources and constraints in OR management. The mathematic model of such surgery scheduling problem with multiple objectives of minimizing makespan, minimizing overtime and balancing resource utilization is proposed. Due to the combinatorial nature of the problem, the nonlinear constraints involved, and the conflicting objectives considered, a Pareto set-based ACO algorithm by aiming at achieving sub-optimal solutions is proposed in this paper.

A Pareto sets based ant colony algorithm with corresponding ant graph, pheromone setting and update, and Pareto sets construction is proposed to solve the multi-objective surgery scheduling problem. A two-layer nested ACO structure as well as the relative mechanisms (i.e. the SMSP pheromone definition, the pheromone updating strategy, and the Pareto set solution) is presented. The ant travel graph is designed as an outer surgery graph and multiple inner resource graphs. We emphasized both the information related to the good solution in makespan and the balanced utilization of the resources. The global (iteration-best) and local (on arc-step completion) pheromone update rules are adopted.

A test case from MD Anderson Cancer Center is collected to validate our method. It provides a total 28 cases in 17 ORs to be staffed 23 RNs and 14 scrubs within one day. Surgeons and nurses are defined with specialty, role, qualification and availability constraints. The scheduling result of the proposed Pareto Set-based ACO algorithm is compared with the real life manual surgery scheduling result, and from the basic ACO approach with single objective of makespan. Four measurements, i.e. end time, individual nurse's maximum overtime, total overtime of nurses and the variation coefficient of working time of resource, are evaluated. Comparison results indicate a superior performance for the proposed Pareto set-based ACO in general. It can be concluded that the algorithm can solve the multiple objective surgery scheduling problem effectively within a reasonable calculation time, while at the same time provide a shortening end time and a relative balanced resource allocations. Future research will be in the direction of extending our ACO algorithm to solving the surgery scheduling problems with uncertainties and more realistic constraints arise in actual OR management in hospital, like surgeons/nurses preference constraints in medical team.

\section{CONFLICT OF INTEREST}

The authors confirm that this article content has no conflict of interest.

\section{ACKNOWLEDGEMENTS}

The Project is supported by Zhejiang Provincial Natural Science Foundation of China (LY12G01007), Ningbo Natural Science Foundation (2013A610109) and K.C.Wong Magna Fund in Ningbo University.

\section{REFERENCES}

[1] D. A. Etzioni, J. H. Liu, M. A. Maggard, and C. Y. Ko, "The Aging Population and Its Impact on the Surgery Workforce", Annals of Surgery, vol. 238, pp. 170-177, 2003.

[2] B. Denton, J. Viapiano, and A. Vogl, "Optimization of surgery sequencing and scheduling decisions under uncertainty", Health Care Management Science, vol. 10, pp. 13-24, 2007.

[3] B. Cardoen, E. Demeulemeester, and J. Beliën, "Operating room planning and scheduling: A literature reviewOriginal", European Journal of Operational Research, vol. 201, pp. 921-932, 2010.

[4] S. A. Erdogan, B. T. Denton, J. J. Cochran, L. A. Cox, P. Keskinocak, J. P. Kharoufeh, and J. C. Smith, "Wiley Encyclopedia of Operations Research and Management Science" John Wiley \& Sons, Denton, 2010.

[5] F. Guerriero, and R. Guido, "Operational research in the management of the operating theatre: a survey", Health Care Management Science, vol. 14, pp. 89-114, 2011.

[6] J. H. May, W. E. Spangler, D. P. Strum, and L. G. Vargas, "The Surgical Scheduling Problem: Current Research and Future Opportunities", Production and Operations Management, vol. 20, pp. 392-405, 2011

[7] S. Ogulata, and R. Erol, "A Hierarchical Multiple Criteria Mathematical Programming Approach for Scheduling General Surgery Operations in Large Hospitals", Journal of Medical Systems, vol. 27, pp. 259-270, Jun 2003.

[8] B. Cardoen, E. Demeulemeester, and J. Belien, "Optimizing a multiple objective surgical case sequencing problem", International Journal of Production Economics, vol. 119, pp. 354-366, Jun 2009. 
[9] J. Beliën, E. Demeulemeester, and B. Cardoen, “A decision support system for cyclic master surgery scheduling with multiple objectives", Journal of Scheduling, vol. 12, pp. 147-161, Apl 2009.

[10] S. V. Jeric, and J. R. Figueira, "Multi-objective scheduling and a resource allocation problem in hospitals", Journal of Scheduling, vol. 15, pp. 513-535, Oct 2012.

[11] W. Xiang, J. Yin, and G. Lim, "Modified ant colony algorithm for surgery scheduling under multi-resource constraints", Advances in Information Sciences and Service Sciences, vol. 5,no. 9, pp. 810818, 2013.
[12] J. Yin, and W. Xiang," Ant Colony Algorithm for Surgery Scheduling Problem.", Lecture Notes in Computer Science, vol. 7331, pp. 198-205, 2012.

[13] M. Dorigo, and T. Stützle, "Ant Colony Optimization" MIT Press, Cambridge, 2004.

[14] A. Mobasher, G. Lim, J.F. Bard, and V. Jordan, "Daily scheduling of nurses in operating suites", IIE Trans actions on Healthcare Systems Engineering, vol. 1, pp. 232-246, 2011.

(C) Wei and Lim; Licensee Bentham Open.

This is an open access article licensed under the terms of the (https://creativecommons.org/licenses/by/4.0/legalcode), which permits unrestricted, noncommercial use, distribution and reproduction in any medium, provided the work is properly cited. 